\title{
THE INFRARED SPECTRA OF SYNTHESIZED AMORPHOUS SILICATES WITH COMPOSITIONS OF OLIVINE AND PYROXENE
}

\author{
Chiyoe Koike[1] and Akira Tsuchiyama[2] \\ [1] Kyoto Pharmaceutical University, \\ Yamashina, Kyoto 607, Japan. \\ [2] Osaka University, Toyonaka, \\ Osaka 560, Japan.
}

\begin{abstract}
Amorphous olivines synthesized by evaporation method show two very broad bands at $10-11 \mu \mathrm{m}$ and $17.5-19 \mu \mathrm{m}$, which resemble the spectra of symbiotic stars. On the other hand, amorphous pyroxenes produced by the same method show two broad bands at 9.5-10.3 $\mu \mathrm{m}$ and 20-22 $\mu \mathrm{m}$, which are narrower than that of amorphous olivine. The features of amorphous olivine were easily altered by heating or hydration, and the peak wavelength of $18 \mu \mathrm{m}$ band was easily shifted to longer wavelengths.
\end{abstract}

\section{INTRODUCTION.}

Recently, many unusual spectra of stars were reported from the IRAS data in the low-dispersion spectra(LRS). For instance, very broad features of $10 \mu \mathrm{m}$ and $18 \mu \mathrm{m}$ bands were found on symbiotic systems. These features are not explained based on the data measured previously in amorphous silicates, because the width of these bands is significantly narrower than those observed in the stars. Here, we report about the simulation of the infrared spectra of symbiotic stars and very unstable characteristics of the $18 \mu \mathrm{m}$ band by alteration.

2. SYNTHESIS OF AMORPHOUS SILICATES.

\subsection{Experiments}

Our experiments are processed in two stages, that is, synthetic experiments and alteration. We synthesized amorphous silicates by an evaporation method using $W$ and Mo boats. Samples were heated at about 1800-2000 ${ }^{\circ} \mathrm{C}$ for several minutes in vacuum. The condensates were obtained on Al substrates several $\mathrm{cm}$ apart from the evaporation source. Starting materials of silicates are olivine with different compositions $\left(\mathrm{FO}_{180}, \mathrm{FO}_{9}, \mathrm{FO}_{80}\right), \mathrm{Ca}-\mathrm{poor}$ and $\mathrm{Ca}-\mathrm{rich}$ pyroxenes.

\subsection{Results and discussion}

The condensates from olivine have almost the olivine stoichiometry, while those from pyroxene are enriched in $\mathrm{SiO}_{2}$. All the condensates contain $W$ or Mo due to contaminations from the boats. These $W$ and Mo are

A.C. Levasseur-Regourd and H. Hasegawa (eds.), Origin and Evolution of Interplanetary Dust, 95-98. (1) 1991 Kluwer Academic Publishers, Printed in Japan. 


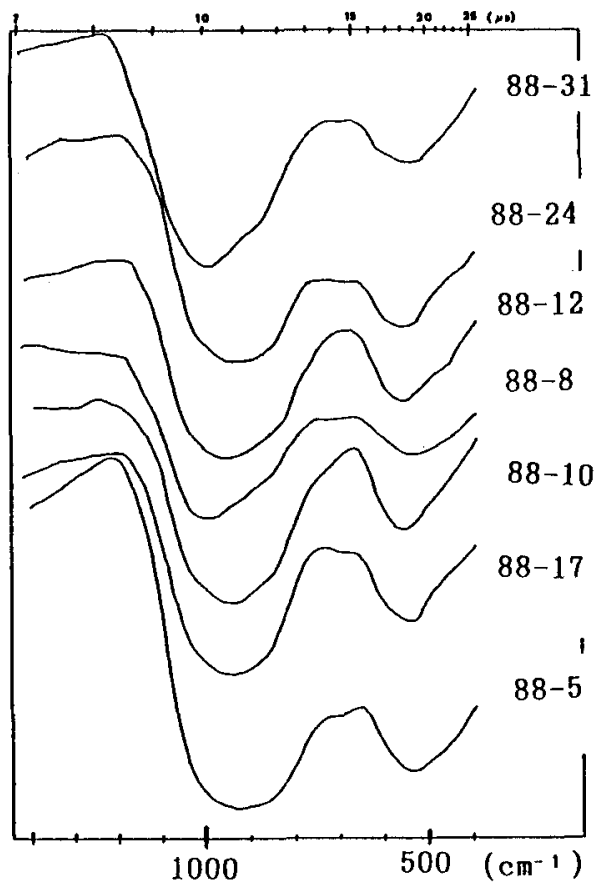

Fig.1. The spectra of amorphous olivines.

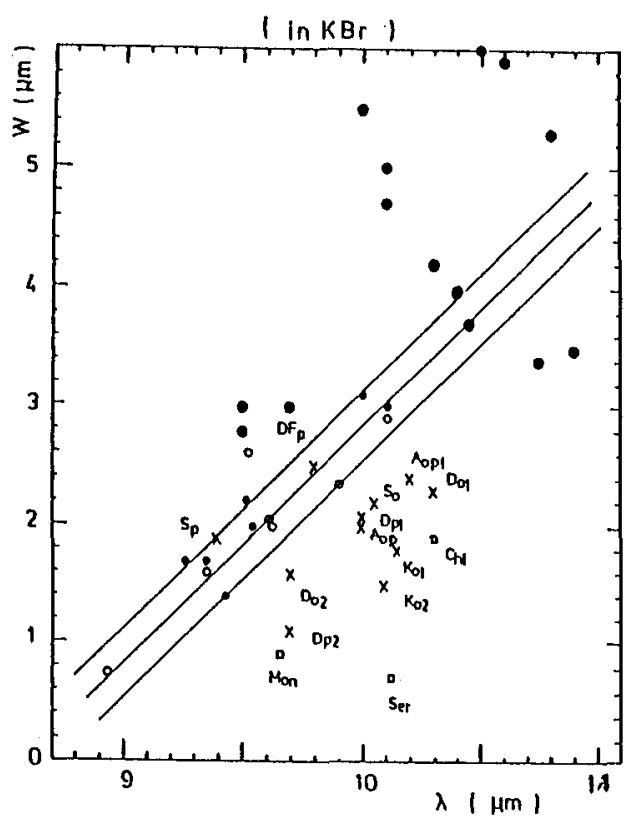

$\mid$ in KBr $\mid$

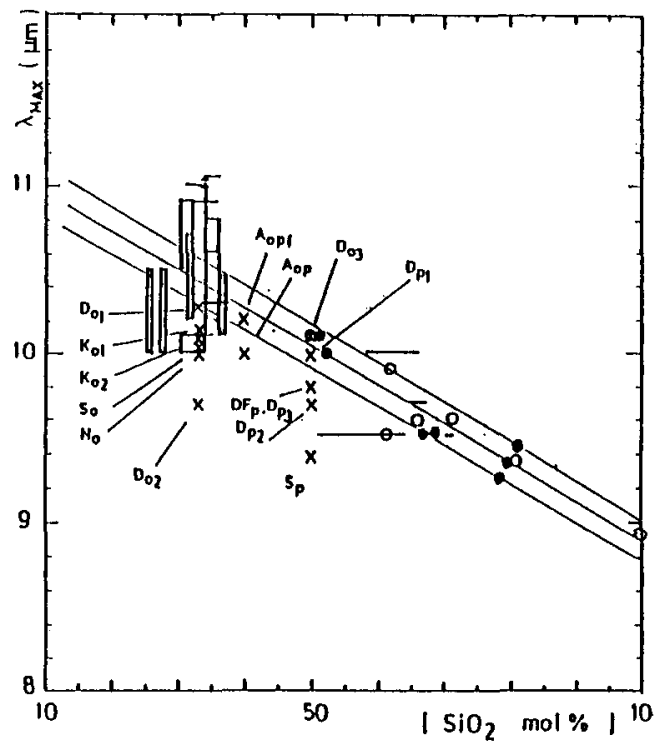

Fig.2. Correlation between the $10 \mu \mathrm{m}$ peak wavelength $\lambda_{\mathrm{m}}$ and $\mathrm{SiO}_{2}$ content. Solid lines and squares are this work, - natural amorphous silicates, o synthetic and fused silictates, $x$ other laboratory data of synthetic amorphous silicates.

Fig.3. Correlation between the $10 \mu \mathrm{m}$ band width $\mathrm{W}$ and $\mathrm{SiO}_{2}$ content. this work, other notations are the same as in Fig.2. 
considered to be metallic based on $X$-ray and electron diffraction patterns. Therefore, the effect of $W$ or Mo to the IR spectra can be ignored. In fact, oxides of $W$ or Mo were not observed in the IR spectra. The infrared spectra of amorphous olivine show two broad bands at wavelengths of about $10-11 \mu \mathrm{m}$ and 18-19 $\mu \mathrm{m}$ (Fig.1), while those of amorphous pyroxene show two broad bands at about 9.5 and $21.5 \mu \mathrm{m}$. The correlation of the $\mathrm{SiO}_{2}$ content with the peak wavelength of $10 \mu \mathrm{m}$ bands is consistent with the previous relation (Koike et al.1987)(Fig.2). However, the band widths are much broader than the other amorphous silicates (Fig.3). For the $18 \mu \mathrm{m}$ bands, amorphous olivine shows an absorption peak at about $18 \mu \mathrm{m}$, but amorphous pyroxene shows a peak at about $22 \mu \mathrm{m}$. The ratio of 10 and $18 \mu \mathrm{m}$ bands of the present samples are lower than in previous data.

These infrared spectra in $\mathrm{KBr}$ are reduced to the spectra in vacuum using dispersion equations to compare with observations. For the first approximations, the corrected features of the amorphous olivine are similar to those of symbiotic stars.

\section{ALTERATION OF THE AMORPHOUS SILICATES}

\subsection{Heating}

We examined changes in the spectra of the above amorphous silicates by annealing and hydration. The amorphous silicates were heated to 150 $600 \mathrm{C}$ for $2-105.25 \mathrm{hr}$. As shown in Fig.4, the spectra of amorphous olivine were not changed up to about $500 \mathrm{c}$, but begun to change distinctly at about $600 \mathrm{c}$. The width of the $10 \mu \mathrm{m}$ band became wider and the peak wavelengths of $18 \mu \mathrm{m}$ band shifted to longer wavelengths. The spectra changed to those of crystal olivine by longer heating at 600 c.

\subsection{Hydration}

The hydration effects were examined by keeping the samples in saturated vapor, in water and in boiling water. The spectrum was sligthtly changed in saturated vapor: a subtle dip appeared at $10 \mu \mathrm{m}$, and the peak wavelength of $18 \mu \mathrm{m}$ band shifted from $18 \mu \mathrm{m}$ to nearly $20 \mu \mathrm{m}$. By keeping the samples in water at room temperature for $42 \mathrm{hr}$, the width of the 10 $\mu \mathrm{m}$ band became slightly narrower and a small shoulder appeared at about $11.8 \mu \mathrm{m}$ (Fig.5). The peak wavelength of the $18 \mu \mathrm{m}$ band was significantly shifted from $18 \mu \mathrm{m}$ to $22 \mu \mathrm{m}$. By keeping the sample in boiling water for $10 \mathrm{hr}$, the spectrum was substantially changed (Fig.5). For this spectrum, the $10 \mu \mathrm{m}$ band showed sharper structures, especially a clear shoulder at $11.5 \mu \mathrm{m}$. The peak wavelength of the $18 \mu \mathrm{m}$ band was also shifted to $22 \mu \mathrm{m}$. From these alteration experiments, we conclude that the $18 \mu \mathrm{m}$ band is very unstable by annealing and hydration.

\section{REFERENCES}

B.G.Anandarao,A.R.Taylor and S.R.Pottasch, (1988) 'Dust emission from symbiotic stars: an interpretation of IRAS observations.', Astron. 


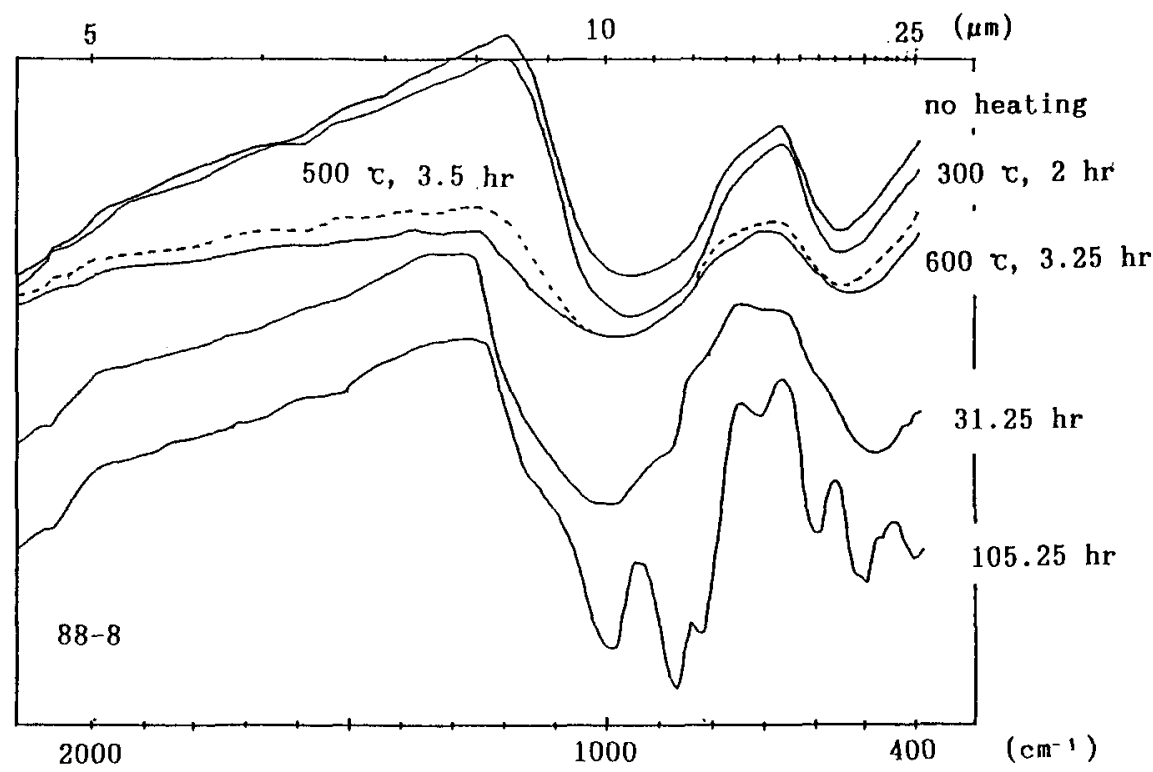

Fig.4. Infrared spectra of an annealed amorphous alivines.

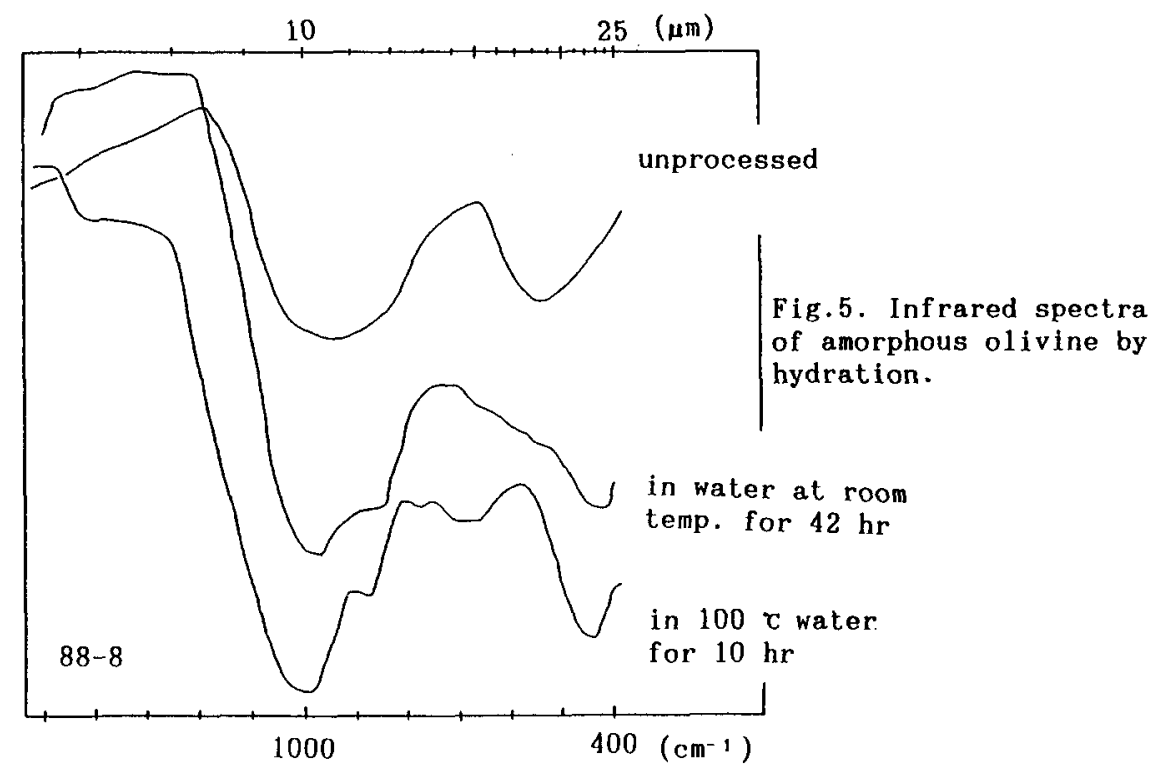

Astrophys. 203, 361-366.

C.Koike and H.Hasegawa, (1987)'Mid-infrared extinction coefficients of amorphous silicates', Astrophys. Space Sience 134,361-379. 